\title{
Dimensiones de la ideología política. Un abordaje operativo desde la Teoría de Respuesta al Ítem
}

\author{
Silvina Brussino; Daniela Alonso; Marcos Cupani; Débora Imhoff; Pamela Paz García; Hugo H. Rabbia
}

Cómo citar este artículo:

Brussino, S., Alonso, D., Cupani, M., Imhoff, D., Paz García, P., \& Rabbia, H.H. (2021). Dimensiones de la ideología política. Un abordaje operativo desde la Teoría de Respuesta al Ítem. Acta Colombiana de Psicología, 24(2), 9-22. https:// www.doi.org/10.14718/ACP.2021.24.2.2

Recibido, octubre 21/2020; Concepto de evaluación, marzo 15/2021; Aceptado, abril 21/2021

\author{
Silvina Brussino \\ ORCID: http://orcid.org/0000-0002-1087-644X \\ Consejo Nacional de Investigaciones Científicas y Técnicas (CONICET), Argentina. \\ Universidad Nacional de Córdoba, Córdoba, Argentina. \\ Daniela Alonso \\ ORCID: http://orcid.org/0000-0003-2080-9571 \\ Consejo Nacional de Investigaciones Científicas y Técnicas (CONICET), Argentina. \\ Marcos Cupani \\ ORCID: https://orcid.org/0000-0003-2132-5552 \\ Consejo Nacional de Investigaciones Científicas y Técnicas (CONICET), Argentina. \\ Universidad Nacional de Córdoba, Córdoba, Argentina. \\ Débora Imhoff \\ ORCID: http://orcid.org/0000-0002-2276-1893 \\ Consejo Nacional de Investigaciones Científicas y Técnicas (CONICET), Argentina. \\ Universidad Nacional de Córdoba, Córdoba, Argentina. \\ Pamela Paz García \\ ORCID: https://orcid.org/0000-0001-9926-3617 \\ Consejo Nacional de Investigaciones Científicas y Técnicas (CONICET), Argentina. \\ Hugo H. Rabbia \\ ORCID: https://orcid.org/0000-0001-9241-5155 \\ Consejo Nacional de Investigaciones Científicas y Técnicas (CONICET), Argentina. \\ Universidad Católica de Córdoba, Córdoba, Argentina.
}

\section{Resumen}

\begin{abstract}
A pesar de que la ideología política es un constructo central para la psicología política contemporánea, en la literatura persisten desacuerdos respecto de su estructura y operacionalización. Teniendo esto en cuenta, el presente trabajo evalúa las propiedades psicométricas de la Escala de Ideología Política de forma operativa, es decir, contemplando los posicionamientos de las personas sobre temas políticos. Para ello, se tomó una muestra probabilística de la población de Córdoba, Argentina $(N=444)$ y se testearon las propiedades psicométricas de esta escala por medio de la teoría de respuesta al ítem. Como resultado, se encontró una estructura bidimensional con dos ejes independientes - conservadurismo y progresismo- cada uno con propiedades de intervalo y ajuste del modelo satisfactorio. Como indicador de validez predictiva, los resultados de la relación de las dimensiones de ideología política con los valores sociales fueron consistentes con la evidencia empírica antecedente. En suma, si bien las propiedades psicométricas de la Escala de Ideología Política se seguirán estudiando, esta es un aporte significativo por la escasez de instrumentos de evaluación que la comprendan de un modo operativo, multidimensional y ecológicamente válido.

Palabras clave: ideología política, conservadurismo, progresismo, teoría de respuesta al ítem.
\end{abstract}

1 Instituto de Investigaciones Psicológicas [CONICET-UNC], Facultad de Psicología, Universidad Nacional de Córdoba. Bv. de La Reforma esq. Enfermera Gordillo. Ciudad Universitaria, Córdoba. Argentina. CP 5000. silvina.brussino@unc.edu.ar

La presente investigación fue financiada por la Agencia Nacional de Promoción de la Investigación, el Desarrollo Tecnológico y la Innovación (PICT2012-2443). 


\title{
Dimensions of political ideology. An operational approach from Item Response Theory
}

\begin{abstract}
Although political ideology is a core concept for contemporary political psychology, divergences persist in the literature regarding its structure and operationalization. This paper assesses the psychometric properties of the Escala de Ideología Politica [Political Ideology Scale] operationally, that is, considering people's positioning regarding different political issues. For this purpose, a probability sample was taken from the population of Córdoba, Argentina $(\mathrm{N}=444)$ and the psychometric properties of this scale were tested by applying Item Response Theory. A two-dimensional structure with two independent axes -Conservatism and Progressivism- was found, each with interval properties and satisfactory model fit. Furthermore, political ideology was correlated with social values, in a way that is consistent with the previous empirical evidence. This provides evidence of predictive validity. In sum, although the psychometric properties of the Political Ideology Scale will be further studied, this paper represents a significant contribution, due to the scarcity of ecologically valid instruments of operational ideology.

Keywords: political ideology, conservatism, progressivism, item response theory.
\end{abstract}

\section{Introducción}

La ideología política (en adelante IP) es uno de los tópicos más debatidos en el campo de la psicología política contemporánea, especialmente en un escenario sociopolítico cambiante y polarizado como el actual (McCoy et al., 2018). Sin embargo, se trata de un constructo no exento de controversias tanto en sus definiciones conceptuales como en sus abordajes instrumentales.

De modo general, entendemos la IP como un sistema de creencias sobre cómo debería ser el orden social (Brussino et al., 2017). Se trata de una configuración de ideas y actitudes con cierto grado de interdependencia y compartidas en alguna medida por el grupo de pertenencia. Por una parte, a nivel sistémico, la IP funciona como organizador social del espacio político y presenta un marco de referencia para la comunicación y posicionamiento de los actores políticos en la escena pública (Freire \& Kivistik, 2013); y por otro, a nivel individual, constituye un heurístico que permite a la ciudadanía reducir la complejidad del universo político y facilita la toma de decisiones (Lau \& Redlawsk, 2006).

Dentro de este marco conceptual, existen diferentes propuestas respecto a la forma en que se estructuran los posicionamientos ideológicos de las personas que redundan en aproximaciones empíricas diferentes. Por un lado se identifican los abordajes simbólicos de la IP que, por su sencillez, son los más aplicados en el campo (Morgan \& Wisneski, 2017). Específicamente, la IP simbólica refiere a la forma en que las personas se autoposicionan en un eje que va de izquierda a derecha, o de progresista/liberal a conservador, por lo cual la IP se puede entender como un constructo unidimensional en un eje bipolar. Por su parte, las dimensiones operativas de la IP se asientan en posicionamientos actitudinales concretos respecto a diversos temas relevantes en la agenda política (Brussino et al., 2017). No obstante, es posible que la ciudadanía aplique distintos marcos ideológicos para la evaluación de diferentes temas, razón por la cual se destaca la multidimensionalidad de este constructo (Morgan \& Wisneski, 2017).

De hecho, la evidencia proveniente de aproximaciones simbólicas a la IP sugiere que un único indicador resulta insuficiente para comprender la complejidad de las orientaciones ideológicas de las personas, especialmente en el ámbito de democracias multipartidistas donde los clivajes ideológico-partidarios están menos estructurados (Brussino et al., 2011). En consecuencia, resulta necesario profundizar respecto a los abordajes operativos de la IP que permitan evaluar su estructuración e identificar su multidimensionalidad, sobre la cual no existe acuerdo en la literatura vigente (Azevedo et al., 2019; Bauer et al., 2017).

En esta línea, algunos trabajos distinguen las dimensiones socioculturales de las dimensiones económicas de la IP, que tendrían tanto correlatos motivacionales como consecuencias actitudinales y comportamentales diferenciales (Costello \& Lilienfeld, 2020). Por ejemplo, Crawford et al. (2017) comprobaron en población estadounidense que la relación entre la IP y el prejuicio hacia distintos grupos sociales variaba en función de dimensiones sociales y económicas: personas conservadoras en temas sociales manifestaban mayor prejuicio hacia grupos liberales en lo social, y viceversa. Sin embargo, la evidencia empírica que permite diferenciar estas dimensiones de la IP no siempre es 
consistente. De otro lado, Azevedo et al. (2019) encuentran que las dimensiones sociales, culturales y económicas están relacionadas y organizadas en un sistema ideológico coherente incluso - aunque en menor medida - en ciudadanos con baja sofisticación política.

Por su parte, Federico y Schneider (2007) señalan que no solo la expertise, sino también variables motivacionales podrían explicar las desviaciones de la estructuración bipolar de la IP, es decir, los casos en que conservadurismo y progresismo no correlacionan negativamente entre sí. Asimismo, Kalmoe (2020) da cuenta de que solo un porcentaje minoritario de la población estadounidense sostiene orientaciones ideológicas estables y coherentes, aspecto que se relaciona con el nivel de conocimiento político. En este punto, resulta relevante considerar que el número de dimensiones de la IP que las personas usen puede variar entre ellas en función de variables individuales - como la sofisticación política - y culturales - como las características del sistema de partidos local-(Morgan \& Wisneski, 2017). Considerando esta circunstancia clave en la investigación empírica del constructo, es posible que no podamos arribar a una estructura universal de la IP, sino que debamos enfatizar en instrumentos ecológicamente válidos, sensibles a las particularidades de cada cultura política.

En esta línea, Lameris et al. (2018) evaluaron operativamente la IP en una muestra holandesa a partir de 40 afirmaciones sobre temas políticos, con lo cual encontraron un modelo de cuatro dimensiones temáticas: apertura, igualdad económica, autodeterminación y eficiencia. $Y$ en un abordaje similar, en Argentina, se desarrolló un instrumento que, a partir de 22 afirmaciones sobre temas relevantes de la política actual planteados en función de la intervención/no intervención del Estado, se identificó cuatro dimensiones operativas de la IP:(a) conservadurismo sexual religioso, que recupera posicionamientos sobre educación sexual, derechos sexuales y (no) reproductivos y la relación IglesiaEstado; (b) progresismo multiculturalista, que remite a la valoración de la injerencia estatal en temas ambientales, diversidad cultural y comunicacional, y consumo responsable de drogas; (c) conservadurismo represivo nacionalista, que refiere a temas de seguridad interna, drogas y respeto a símbolos nacionales; y (d) progresismo garantista, que remite a la equidad y justicia en el acceso a servicios públicos y garantías para la expresión de protesta (Brussino et al., 2011). En este caso, las dimensiones económicas de la IP tuvieron un papel secundario en comparación con temas sociales y culturales.

Una posible explicación de esta variación intra e interindividual en la estructuración de la IP redunda en que las técnicas clásicas para los análisis psicométricos de los instrumentos de evaluación resulten, en ocasiones, insuficientes. De hecho, autores como Morgan y Wisneski (2017) señalan que la aplicación de análisis factoriales clásicos para la identificación de la estructura subyacente a grandes muestras puede no ser la estrategia adecuada para abordar un constructo con gran variación interindividual. En función de ello, consideramos relevante no solo trabajar en la actualización de las dimensiones analíticas de la IP, procurando una mayor validez ecológica, sino también aplicar técnicas analíticas más adecuadas. Es por ello que en este trabajo proponemos analizar la Escala de IP a partir de la aplicación de la Teoría de Respuesta al Ítem (TRI), un modelo psicométrico que permite obtener medidas invariantes, independientemente de los instrumentos utilizados y las personas evaluadas, así como calcular el error de medida para cada ítem y persona (Engelhard Jr., 2013).

En la actualidad, la TRI y el modelo de Rasch, en particular, son abordajes populares para la creación y validación de instrumentos, y, entre los modelos alternativos de TRI, el modelo de Escala de Clasificación (MEC) se ajusta a las características de la variable IP. En particular, el MEC es un modelo politómico que se deriva del modelo de Rasch, $\mathrm{y}$ que tiene como supuestos: (a) la unidimensionalidad, es decir, que todos los ítems miden el mismo constructo; (b) la independencia local de ítems y personas, que implica que la respuesta de una persona a un ítem no se ve afectada por su respuesta a otro ítem, lo cual aplica también a los ítems; y (c) la discriminación homogénea de los elementos, que implica que todos los elementos tienen igual capacidad de discriminación (Andrich, 1978).

Ahora bien, para completar el análisis psicométrico de la IP, también se planificaron estudios de validez predictiva. Para ello, estimamos la correlación entre las dimensiones de la IP y los valores sociales (vs), que se definen como metas motivacionales u objetivos deseables, de carácter transituacional, que expresan necesidades materiales y sociales básicas (Schwartz, 2003). Aunque relativamente más estables que las orientaciones ideológicas, los vs tienen también un rol en la organización del conocimiento y preferencias en relación con lo político (Cordero García, 2008): conforman estructuras normativas subyacentes que orientan los posicionamientos de las personas sobre temáticas sociopolíticas diversas.

Numerosos estudios han documentado la relación e interacción entre ambas dimensiones analíticas (valores de IP) (Brussino et al., 2017; Caprara et al., 2017, 2018; Sheldon \& Nichols, 2009), y la mayoría de estos utiliza el continuo izquierda-derecha o liberal-conservador para testear la relación entre los dos conceptos. Específicamente, se ha podido establecer que la izquierda se asociaría a valores de igualdad - luchas en favor de la clase trabajadora, eliminación de privilegios, desigualdades y pobreza - y la 
preferencia por el cambio social, mientras que la derecha se sustentaría en valores de libertad —énfasis en libertades individuales, sostenimiento de jerarquías y privilegios como parte de un orden natural - y una preferencia por la conservación del statu quo, el orden y la estabilidad (Jost, 2006).

Estas relaciones también fueron encontradas en otros estudios en Argentina (D’Adamo \& García Beaudoux, 2002), donde quienes se posicionaron a la izquierda suscriben más valores asociados a la igualdad en comparación con personas de derecha, quienes acordaban mayormente con el sostenimiento de las jerarquías sociales. En la misma línea, las bipolaridades autopromoción (poder y logro) versus autotrascendencia (universalismo y benevolencia), y apertura al cambio (estimulación y autodirección) versus conservación (seguridad, conformidad y tradición) de la estructura de valores de Schwartz (2003) correlacionan con la oposición clásica de liberalismo versus intervencionismo, y conservadurismo versus progresismo (Caprara et al., 2006; Cordero García, 2008; Sheldon \& Nichols, 2009). Por su parte, Brussino et al. (2013) replicaron la estructura de relaciones esperada entre estas variables, pero encontraron que diferentes dimensiones del progresismo se relacionan con diferentes perfiles axiológicos, donde se observa que los perfiles de las personas conservadoras son más homogéneos.

Finalmente, resulta relevante mencionar que la relación entre valores e IP puede ser sensible a las características de la cultura política local. De ello dan cuenta Piurko et al. (2011) al analizar la relación entre valores personales básicos y la orientación ideológica de ciudadanos de 20 países europeos, a los cuales clasificaron según su trayectoria democrática en liberales, tradicionales y poscomunistas. Sus resultados mostraron, por ejemplo, que la religiosidad era relativamente más importante en las democracias tradicionales, y que en los países poscomunistas — con una historia democrática corta - los significados asociados a la izquierda y la derecha resultaron menos claros y que los patrones de relación con los valores básicos fueron variables.

No obstante, si bien la mayoría de los estudios aplican el inventario de valores desarrollado por Schwartz (2003), en ocasiones se han identificado dificultades para la validación local o regional de la estructura teórica originalmente propuesta (Steinmetz et al., 2012). En consecuencia, para este trabajo se decidió recuperar aportes regionales que proponen un abordaje empírico ecológicamente más ajustado de los vs. De esta manera, la escala escogida, desarrollada en Brasil (Pereira et al., 2004, 2005) y validada en Argentina (Alonso \& Brussino, 2018), propone cuatro sistemas valorativos centrales: materialista, posmaterialista, hedonista y religioso (Pereira et al., 2004, 2005). Acá, por su definición teórica, el sistema de valores materialista es cercano a las dimensiones del poder, y el posmaterialista al universalismo, benevolencia y autodirección; mientras que los valores religiosos se corresponden con motivaciones de conformidad y tradición, y los valores hedonistas con el sistema homónimo de Schwartz y con la estimulación (Pereira et al., 2005). En una aproximación empírica, se encontró evidencia de una relación positiva entre los valores hedonistas y la tolerancia política y negativa con el autoritarismo. Por su parte, los valores religiosos y materialistas tenían un patrón opuesto: los valores religiosos se asociaban negativamente con la tolerancia política y los religiosos negativamente con el autoritarismo (Barros et al., 2009). En consecuencia, se espera que los sistemas religioso y materialista correlacionen positivamente con posicionamientos ideológicos conservadores, y que los sistemas posmaterialistas y - en menor medida - hedonista lo hagan con orientaciones progresistas.

\section{Método}

\section{Tipo de estudio}

La investigación desarrollada corresponde a un estudio de tipo instrumental (Montero \& León, 2007) respecto a la Escala de Ideología Política.

\section{Participantes}

En este estudio participaron 444 personas (el $48.2 \%$ eran varones, el $51.3 \%$, mujeres, y el $0.5 \%$ restante no indicó su sexo) entre 18 y 65 años $(M=42.4, D S=9.14)$ de la ciudad de Córdoba, Argentina. Se utilizó un muestreo probabilístico polietápico por conglomerados con probabilidades idénticas (Lohr, 2000). En una primera etapa se seleccionaron radios censales de manera aleatoria; luego, dentro de los radios censales se seleccionaron por el mismo procedimiento hogares y hogares de reemplazo - para el caso de personas que no accedieran a responder el cuestionario - En cada hogar seleccionado se contactaba a personas mayores de 18 años, y solo podía responder al cuestionario una persona por hogar. El tamaño de la muestra fue estimado para una población infinita, asumiendo un nivel de confianza del $95 \%$ y $\pm 5 \%$ de margen de error.

\section{Instrumentos}

\section{Escala de Ideología Politica}

Se utilizó una versión revisada de la escala elaborada por Brussino et al. (2011). La escala original consta de 
22 ítems situacionales sobre tópicos específicos (política social, economía, sexualidad, drogas, religión, seguridad, comunicación, medio ambiente y nacionalismo) en función del eje intervención/no intervención del Estado. Las opciones de respuesta se dan en una escala tipo Likert de 5 puntos - de 1 = "totalmente en desacuerdo" a 5 = "totalmente de acuerdo"- En un estudio con población similar a la de este trabajo (Brussino et al., 2011), estos ítems se distribuyeron en cuatro subescalas $(\mathrm{KMO}=, 83, \mathrm{sig} .=.000)$ que explicaron el $45 \%$ de la varianza. Cada subescala se conformó a partir de la sumatoria del puntaje de cada uno de sus ítems: conservadurismo sexual religioso ( 7 ítems, $\alpha=.77$ ), progresismo multiculturalista (7 ítems, $\alpha=.71$ ), conservadurismo represivo nacionalista ( 5 ítems, $\alpha=.70$ ), y progresismo garantista ( 3 ítems, $\alpha=.66$ ). En el presente estudio se crearon nueve ítems adicionales (en cursivas en el Anexo) que responden a temas relevantes en el contexto específico de aplicación — por ejemplo, política pública sobre regulación estatal de medios de comunicación masiva- Para la construcción de estos ítems se utilizó el mismo procedimiento que para la construcción de los ítems originales (Brussino et al., 2011). Así, el instrumento procura incrementar su validez ecológica.

\section{Escala de Valores Sociales}

Se aplicó la versión adaptada localmente (Alonso \& Brussino, 2018) de la escala desarrollada por Pereira et al. (2004), que consta de una lista de 22 valores para evaluar cuatro sistemas valorativos: (a) materialista -4 ítems (b) hedonista -4 ítems - , (c) religiosidad -4 ítems - y (d) posmaterialista - 10 ítems - Las opciones de respuesta van de 1 a 10 en función de la importancia que atribuye el/la participante a cada valor, pensando en el desarrollo de una sociedad ideal. El puntaje de cada subescala se construyó con la sumatoria de cada uno de los ítems que la conforman. Los índices de confiabilidad de cada subescala fueron: $\alpha$ de Cronbach de .76 para los valores materialistas, .77 para los valores hedonistas, .89 para los valores de religiosidad, y .77 para los valores posmaterialistas.

\section{Procedimiento}

Los instrumentos de recolección de datos fueron administrados de forma domiciliaria, individual y oral por miembros del equipo de investigación debidamente entrenados/as. Durante todas las instancias se garantizó el cumplimiento de las normas éticas para la investigación (APA, 2010), de manera que se enfatizó en la naturaleza voluntaria de la participación en el estudio y se solicitó a los/as participantes su consentimiento informado. Además, se garantizó el anonimato y la confidencialidad en el manejo de los datos. El porcentaje de rechazo a participar fue de un $8 \%$.

\section{Análisis de datos}

Se realizó un análisis factorial exploratorio para identificar la estructura factorial más adecuada con el programa FACTOR 9.3. Para determinar el número de dimensiones se utilizó la Implementación Óptima del Análisis y el Mínimo Promedio Parcial. Las soluciones iniciales se obtuvieron mediante el uso de una estimación robusta de mínimos cuadrados no ponderados (RULS) con estadísticos de ajuste con corrección media, tal como se implementó en el programa FACTOR (Lorenzo-Seva \& Ferrando, 2006). La evaluación del ajuste de los datos del modelo se basó en el error cuadrático medio de aproximación (RMSEA) como medida de ajuste aproximado; el índice de bondad de ajuste (GFI) como medidas absolutas de ajuste; y el índice de ajuste no normado (NNFI) como medida relativa de ajuste con respecto al modelo de independencia nula. El método de rotación de la solución factorial resultante fue Robust Promin (Lorenzo-Seva \& Ferrando, 2019). También se estimó la confiabilidad denominada Orion (Ferrando \& Lorenzo-Seva, 2016) y el coeficiente Omega (McDonald, 1999) para cada dimensión obtenida del AFE.

Luego, se analizaron las dimensiones resultantes desde el Modelo de Rasch, utilizando el software RUMm2030 (Andrich et al., 2010), donde se utilizó el log-likelihood ratio test para establecer qué opción de parametrización se utilizaría. Si se obtienen valores de significación $p<.01$, se verifica que la distancia entre las categorías de respuesta no se mantiene constante a través de los ítems (Tennant \& Conaghan, 2007). También, se examinó el funcionamiento y estructura de las categorías de respuesta. Cuando se corresponden las respuestas de los/as participantes a los ítems, los umbrales se encuentran ordenados y, en consecuencia, los ítems son consistentes con la estimación métrica del constructo medido.

La unidimensionalidad se evaluó utilizando el método propuesto por Smith (2002), que consiste en aplicar el Análisis de los Componentes Principales (PCA) de los residuos, y luego delimitar dos subtest de ítems, uno compuesto por ítems con correlaciones positivas y otro con correlaciones negativas $( \pm .30)$. Estos subtests se sometieron luego a un análisis de pruebas $t$ emparejadas para probar si las estimaciones de las personas de cada grupo difieren significativamente de las derivadas de todos los ítems. Para que este supuesto se cumpla, el porcentaje de pruebas por fuera del rango -1.96 a 1.96 no debe exceder el 5 \% (Tennant \& Pallant, 2006). La independencia local se 
evalúo a través de la matriz de correlaciones de los residuos, donde valores $>0.2$ indican presencia de dependencia local (Andrich et al., 2010).

A partir del análisis de interacción ítem-persona, obtuvimos un resumen general de las variaciones en relación con el ajuste al modelo y si los datos concuerdan con lo esperado por el modelo - media cercana a 0 y la desviación estándar cercana a $1-$. Por otro lado, el estadístico residual estandarizado comprendido entre \pm 2.5 indica un buen ajuste al modelo. Además, se utilizó el estadístico de interacción ítem-rasgo que utiliza el chi-cuadrado $\left(\chi^{2}\right)$ para probar la invarianza de la prueba a través del rasgo. Un chi-cuadrado significativo indica que la ubicación relativa de la dificultad de la pregunta no es constante a través del rasgo. Cuando los datos se ajustan al modelo, la interacción ítem-rasgo tiene un valor de $\chi 2$ bajo, con una $p>.05$. El índice de separación persona (PSI) nos informa el grado en el cual el instrumento diferencia a las personas en el constructo que medimos. Un valor de .70 es óptimo para utilizar el instrumento en grupos.

Finalmente, el funcionamiento diferencial de los ítems (DIF) se analizó según el sexo de los/as participantes. Se analizaron dos clases de sesgos: DIF-uniforme — donde se observa en el grupo una diferencia sistemática de las respuestas dadas a un ítem a través del rango que mide el atributo-y DIF-no uniforme - donde no existe uniformidad en las diferencias entre los grupos - por medio de la curva característica del ítem, y se confirmó estadísticamente a partir del ANOva. Por último, para evaluar la validez predictiva de la Escala de Ideología Política, se estimaron las correlaciones bivariadas entre sus dimensiones y las mediciones de valores psicosociales. Como criterio para evaluar el tamaño del efecto de las correlaciones se partió de las indicaciones de Cohen (1988) para la interpretación de la magnitud de tamaños del efecto (pequeños $r=.10$; medio $r=.25$, y grande $r=.40$ ).

\section{Resultados}

\section{Análisis factorial exploratorio}

La medida de adecuación muestral de Kaiser-MeyerOlkin obtenida (.74) y la prueba de esfericidad de Bartlett con valores de $4971.7(d f=465 ; p=.00)$ advirtieron la viabilidad de realizar el análisis factorial. Además, la Implementación Óptima del Análisis Paralelo y el Mínimo Promedio Parcial sugirieron la extracción de dos o tres factores.

En primer lugar, se analizó una estructura de tres dimensiones y se observó que el último factor a extraer estaba compuesto por cinco ítems, pero solo tres de ellos mostraron valores superiores a .40. Esta solución factorial fue considerada no viable para nuestros datos, ya que se aconseja que cada factor tenga por lo menos cuatro ítems con correlaciones iguales o superiores a .40 (Glutting, 2002).

La estructura factorial de dos factores explicó un $32 \%$ de la varianza, y los índices de ajuste ( $\mathrm{RMSEA}=.05, \mathrm{NNFI}=.94$, $\mathrm{GFI}=.95)$ indicaron que la solución de dos factores se ajusta a los datos. Por lo tanto, se decidió retener aquellos ítems con una saturación factorial superior a .40 en un factor y no superior a .30 en los restantes factores. Si un ítem saturaba por encima de .30 en dos factores, se consideró que contribuía al factor en que la saturación era mayor, siempre que la diferencia entre las cargas factoriales del ítem en cuestión fueran mayores que .10. De no ser así, y con el objetivo de retener ítems unidimensionales en cada subescala, el ítem era un candidato para descartar (Morrison et al., 2000).

El primer factor quedó integrado por 13 ítems con pesos factoriales comprendidos entre .40 a .70 pertenecientes a la subescala conservadurismo, donde los índices de confiabilidad para esta escala fueron de Orion $=.85 \mathrm{y}$ el McDonald's ordinal Omega $=.86$; y el segundo factor quedó compuesto por 10 ítems con pesos factoriales entre .42 y .65 , pertenecientes a la subescala progresismo, cuyos índices de confiabilidad fueron de 87 y .84 . De la totalidad de los ítems, tres ítems de la subescala conservadurismo y cinco ítems de la subescala progresismo presentaron cargas factoriales compartidas o con valores por debajo del .30, pero que fueron retenidos para analizarlos mediante el Modelo de Rasch.

\section{Subescala de progresismo}

Para esta subescala, el Log-likelihood Ratio Test fue significativo $\left(\chi^{2}=143.80 ; d f=14 ; p<.00\right)$, por lo cual fue apropiado utilizar el Partial Credit Model, que acepta que la distancia entre los umbrales no sea igual en todos los ítems. El análisis de los umbrales de la escala de cinco categorías de respuestas indicó la presencia en un desorden entre los umbrales. Por lo tanto, las categorías originales colapsaron en categorías adyacentes de diferentes maneras, y la combinación 11344 fue mejor que otras, con lo cual todas las medidas y calibraciones resultaron ordenadas (véase Tabla 1).

La subescala de progresismo presentó una media $(M)$ para los ítems de .00 y una desviación estándar $(D E)$ de .69, y para las personas una $M=.65$ y un $D E=.78$. Además, se comprueba cierta desviación de los ítems en la interacción de un chi-cuadrado significativo $(\chi 2=118.81 ; d f=64$; $p<.00$ ), lo que sugiere desajuste en alguno de los reactivos. En la Tabla 1 se puede advertir que el ítem IP.31 presenta un valor residual superior a $\pm 2.5 \mathrm{y}$ un valor de $\chi^{2}$ que fue 
Tabla 1.

Valores de umbral y su error para cada paso de categoría, parámetro de dificultad y error de medición, e índices de ajuste de los items para la subescala de progresismo

\begin{tabular}{|c|c|c|c|c|c|c|c|c|c|}
\hline \multirow[b]{2}{*}{ Ítem } & \multicolumn{4}{|c|}{ Umbral } & \multicolumn{5}{|c|}{ Ajuste } \\
\hline & $\begin{array}{c}\text { Paso de } 1 \\
\text { a } 2\end{array}$ & $\mathrm{SE}_{1-2}$ & $\begin{array}{c}\text { Paso de } 2 \\
\text { a } 3\end{array}$ & $\mathrm{SE}_{2-3}$ & $\begin{array}{c}\text { Locación } \\
\text { (logits) }\end{array}$ & $\mathrm{SE}$ & Residual & $\chi^{2}$ & $p$ \\
\hline IP.5 & -1.02 & .10 & 1.02 & .10 & -0.99 & .09 & 0.59 & 2.15 & .71 \\
\hline IP.13 & -0.93 & .10 & 0.93 & .11 & -0.07 & .08 & -1.66 & 18.03 & .00 \\
\hline IP. 16 & -0.83 & .10 & 0.83 & .10 & -0.41 & .08 & -0.37 & 7.89 & .10 \\
\hline IP. 17 & -1.15 & .10 & 1.15 & .10 & -0.54 & .08 & -0.58 & 10.03 & .04 \\
\hline IP.21 & -1.09 & .10 & 1.09 & .10 & -0.70 & .09 & -0.50 & 8.98 & .06 \\
\hline IP. 28 & -1.21 & .10 & 1.21 & .11 & -0.19 & .08 & 0.04 & 3.41 & .49 \\
\hline IP. 1 & -0.21 & .10 & 0.21 & .12 & 0.97 & .07 & 1.22 & 7.30 & .12 \\
\hline IP. 6 & -1.16 & .10 & 1.16 & .10 & -0.92 & .09 & 0.41 & 2.79 & .59 \\
\hline IP.9 & -0.83 & .10 & 0.83 & .10 & -0.23 & .08 & 1.40 & 8.63 & .07 \\
\hline IP.11 & -0.34 & .10 & 0.34 & .10 & -0.22 & .07 & 0.12 & 2.62 & .62 \\
\hline IP. 20 & -0.72 & .10 & 0.72 & .11 & 0.36 & .07 & 1.33 & 2.21 & .70 \\
\hline IP. 24 & -0.61 & .10 & 0.61 & .12 & 0.75 & .07 & 2.48 & 10.74 & .03 \\
\hline IP. 25 & -1.45 & .10 & 1.45 & .11 & -0.14 & .09 & -0.75 & 12.82 & .01 \\
\hline IP. 26 & -0.69 & .10 & 0.69 & .11 & 0.15 & .07 & -0.16 & 1.71 & .79 \\
\hline IP.27 & -1.02 & .10 & 1.02 & .12 & 0.72 & .08 & 0.69 & 1.84 & .76 \\
\hline IP.31 & -1.41 & .10 & 1.41 & .18 & 1.45 & .09 & 2.57 & 17.67 & .00 \\
\hline
\end{tabular}

significativo (ajuste de Bonferroni al $\alpha=.05$ ). Cuarenta y dos participantes $(9.48 \%)$ tuvieron valores residuales \pm 2.5 , lo que indica que el modelo propuesto explica un $90.52 \%$ de las respuestas de los/as participantes. El Psi fue de .70, considerado aceptable, y el supuesto de unidimensionalidad se cumplió, ya que el análisis de pruebas $t$ emparejadas quedó fuera del rango \pm 1.96 en un $1.81 \%$. En relación con el supuesto de independencia local se observó que los ítems IP.16 e IP.17, y los ítems IP.1 e IP.26 presentaron correlaciones residuales de .21 y .24 , respectivamente. Por último, no se observó DIF uniforme, como tampoco DIF no uniforme.

Con base en estos resultados, se eliminó el ítem IP.31 (véase Anexo) por presentar un valor residual superior a \pm 2.5 . Al reanalizar los datos sin este reactivo, el modelo identificó al ítem IP.24 con un valor residual superior a \pm 2.5 . En este modelo, se observó que los ítems IP.1 e IP.26 continúan presentando una correlación residual mayor a .20, por lo tanto, se decidió eliminar el ítem IP.26. Es de destacar que el ítem IP.31 es uno de los nuevos ítems desarrollados para este estudio, mientras que los ítems IP.24 e IP.26 corresponden al conjunto de ítems originales.

Finalmente, el modelo resultante quedó conformado por 13 ítems, y en el análisis final se observó una $M=.00$ y una
$D E=.59$ para los ítems, y una $M=.84$ y una $D E=.86$ para las personas, con una distribución de la muestra de -1.38 $\mathrm{a}+3.18$ logits (véase Figura 1). El valor de PSI (.68) indicó un índice de fiabilidad aceptable. Todos los ítems ajustaron al modelo y se cumplió el supuesto de independencia local y de unidimensionalidad.

\section{Subescala de conservadurismo}

En esta subescala, el Log-likelihood Ratio Test fue significativo $(\chi 2=124.29 ; d f=13 ; p<.000)$, el análisis de los umbrales indicó la presencia en un desorden entre los umbrales, y la combinación 11344 fue mejor que otras combinaciones (véase Tabla 2).

En la subescala de conservadurismo la media para los ítems fue de .00 y la desviación estándar de .59 , y para las personas la $M=-.48$ y la $D E=.89$. Se comprueba cierta desviación de los ítems en la interacción de un chi-cuadrado significativo $(\chi 2=206.09 ; d f=60 ; p<.00)$. Los ítems IP.19, IP.29 e IP.30 presentaron valores residuales \pm 2.5 . También, los ítems IP.19 e IP.30 presentaron desajuste y el valor de $\chi 2$ fue significativo (ajuste de Bonferroni al $\alpha .05$ ). Un/a participante presentó valores extremos y $39(8.8 \%)$ un valor residual \pm 2.5 . El PSI fue de .77 , y el supuesto de independencia local se cumplimentó. 
Figura 1.

Mapa Persona-Ítems. Subescala de progresismo

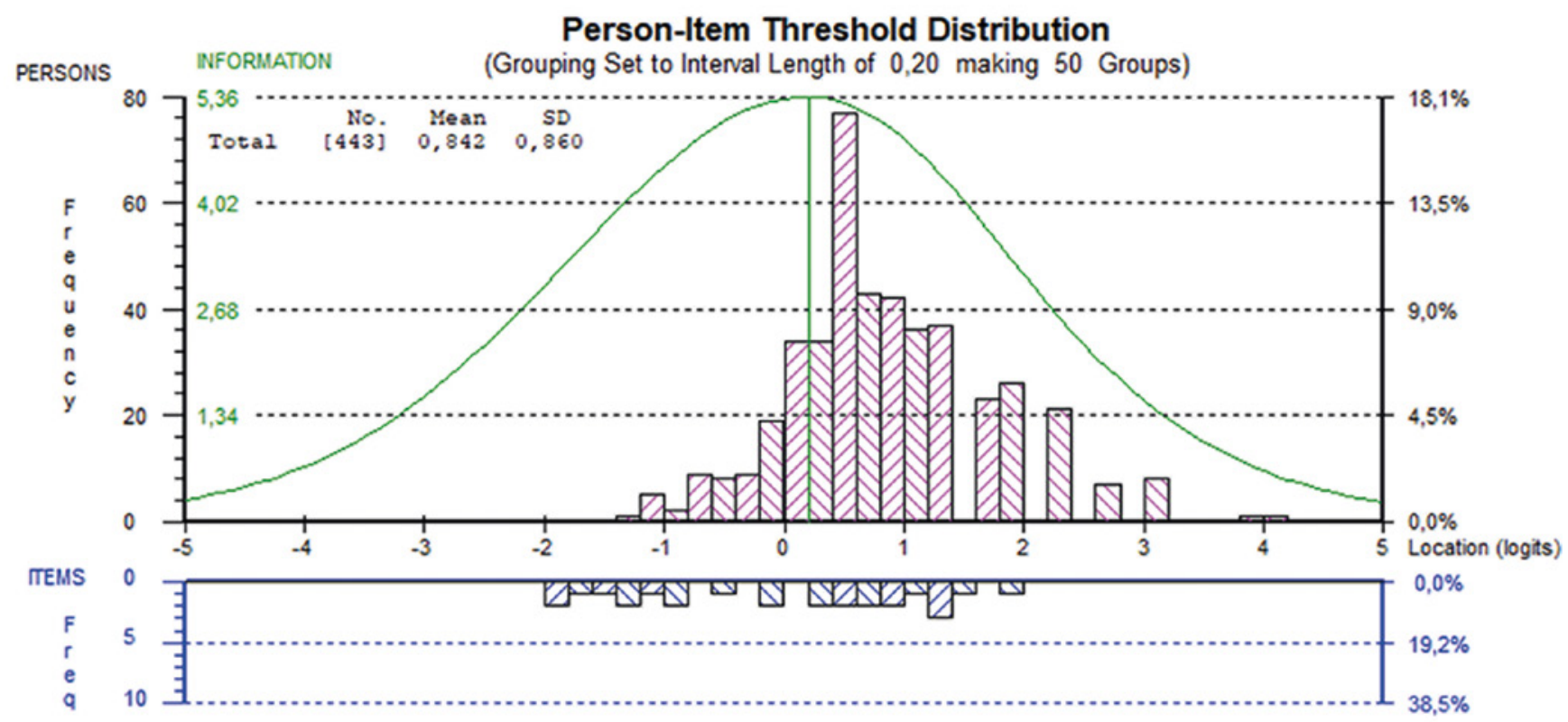

Tabla 2.

Valores de umbral y su error para cada paso de categoría, parámetro de dificultad y error de medición, e indices de ajuste de los items para la subescala de conservadurismo

\begin{tabular}{|c|c|c|c|c|c|c|c|c|c|}
\hline \multirow[b]{2}{*}{ Ítem } & \multicolumn{4}{|c|}{ Umbral } & \multicolumn{5}{|c|}{ Ajuste } \\
\hline & $\begin{array}{c}\text { Paso de } 1 \\
\text { a } 2\end{array}$ & $\mathrm{SE}_{1-2}$ & $\begin{array}{c}\text { Paso de } \\
2 \text { a } 3\end{array}$ & $\mathrm{SE}_{2-3}$ & $\begin{array}{c}\text { Locación } \\
\text { (logits) }\end{array}$ & $\mathrm{SE}$ & Residual & $\chi^{2}$ & $p$ \\
\hline IP. 2 & -0.33 & .10 & 0.33 & .13 & 0.05 & .07 & 1.82 & 2.62 & .62 \\
\hline IP.3 & -0.51 & .10 & 0.51 & .11 & -0.57 & .07 & -0.47 & 3.95 & .41 \\
\hline IP. 4 & -0.38 & .11 & 0.38 & .18 & 0.82 & .08 & -1.45 & 17.54 & .00 \\
\hline IP.7 & -0.45 & .10 & 0.45 & .15 & 0.43 & .08 & -2.45 & 14.83 & .01 \\
\hline IP. 8 & -0.57 & .10 & 0.57 & .16 & 0.55 & .08 & 0.64 & 2.15 & .71 \\
\hline IP. 10 & -0.89 & .10 & 0.89 & .12 & -0.24 & .08 & -0.45 & 5.57 & .23 \\
\hline IP.12 & -1.20 & .10 & 1.20 & .11 & -0.86 & .08 & 0.30 & 4.21 & .38 \\
\hline IP.14 & -0.21 & .11 & 0.21 & .13 & 0.16 & .07 & -1.55 & 8.79 & .07 \\
\hline IP. 15 & -0.84 & .10 & 0.84 & .14 & 0.06 & .08 & -0.25 & 1.84 & .77 \\
\hline IP.18 & -0.82 & .10 & 0.82 & .16 & 0.49 & .08 & -1.81 & 11.50 & .02 \\
\hline IP.19 & -0.83 & .10 & 0.83 & .20 & 0.90 & .09 & -2.50 & 22.70 & .00 \\
\hline IP. 22 & -0.95 & .10 & 0.95 & .15 & 0.21 & .08 & 0.52 & 2.08 & .72 \\
\hline IP. 23 & -1.15 & .10 & 1.15 & .12 & -0.47 & .08 & 0.40 & 5.01 & .29 \\
\hline IP.29 & -1.07 & .10 & 1.07 & .12 & -0.48 & .08 & 2.63 & 9.60 & .05 \\
\hline IP.30 & -0.79 & .10 & 0.79 & .11 & -1.04 & .08 & 6.68 & 93.66 & .00 \\
\hline
\end{tabular}


Figura 2.

Mapa Persona-Ítems. Subescala de conservadurismo

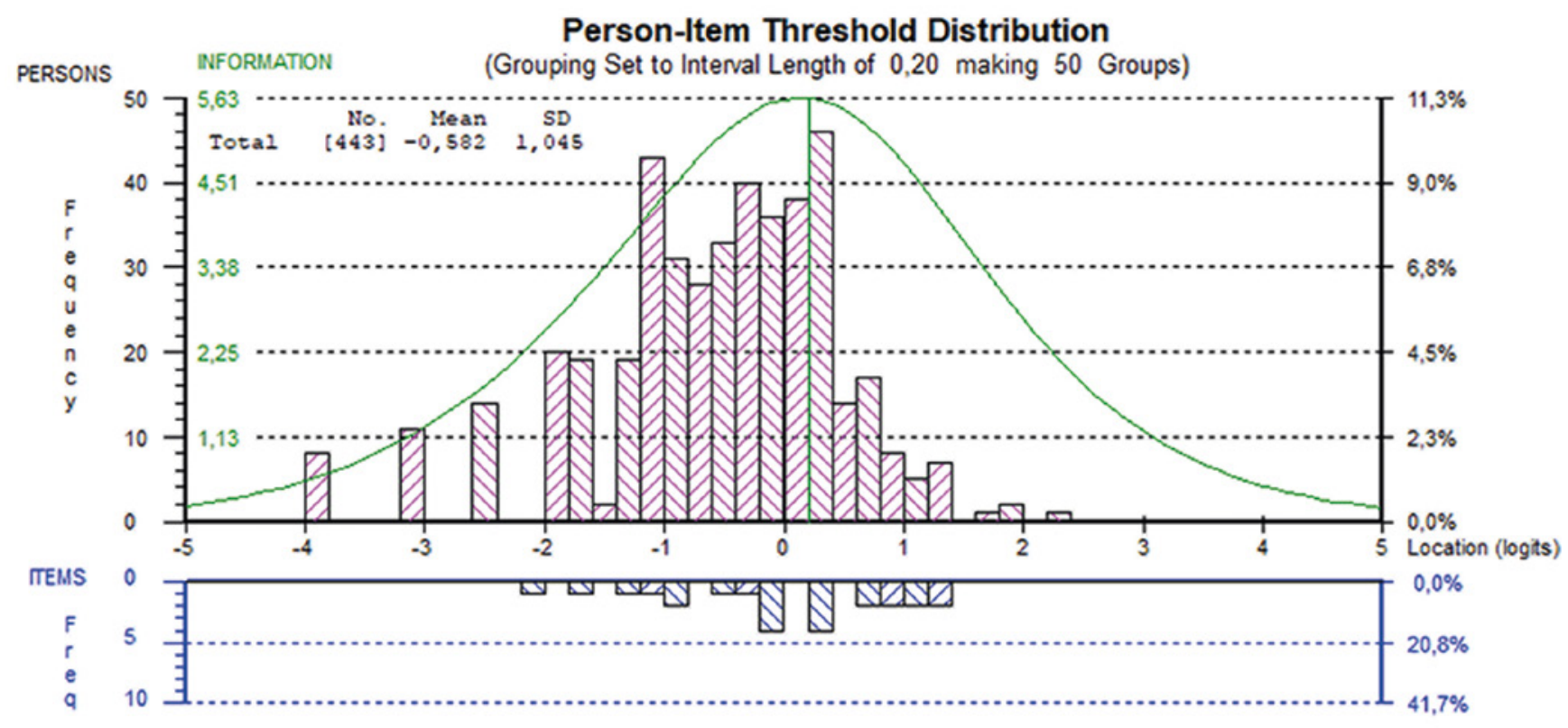

Del mismo modo, el supuesto de unidimensionalidad se cumplió, ya que el análisis de pruebas $t$ emparejadas estuvo fuera del rango \pm 1.96 en un $4.87 \%$. Por último, no se observó DIF uniforme ni DIF no uniforme. Considerando estos resultados, se eliminaron los ítems IP.19 (ítem de la versión original), IP.29 e IP.30 (ítems nuevos). Así, el modelo resultante quedó conformado por 12 ítems, y en el análisis final se observó una $M=.00$ y una $D E=.52$ para los ítems, y una $M=-.58$ y una $D E=1.00$ para las personas, con una distribución de la muestra de -3.02 a +2.31 logits (véase Figura 2). El valor de PSI (.75) indicó un aceptable índice de fiabilidad, todos los ítems ajustaron al modelo, y se cumplió el supuesto de independencia local y de unidimensionalidad.

Evidencia de validez basada en la relación con otras variables

Para establecer la relación entre las dimensiones de la IP y los valores sociales se efectuaron dos análisis complementarios. En primer lugar, se aplicó el estadístico de correlación de Pearson para estimar el nivel de asociación entre las variables (véase Tabla 3). Los resultados indican que las correlaciones observadas variaron entre un tamaño del efecto medio a grande, de acuerdo con la propuesta de Cohen (1988).

Posteriormente, en el segundo análisis complementario se realizó un análisis de regresión múltiple, donde las variables dependientes fueron las dimensiones de la IP, y las variables independientes fueron los valores sociales. Previo a esto, se verificó el cumplimiento de los supuestos para garantizar la validez del procedimiento (Tabachnick \& Fidell, 2001), y luego se incorporaron las variables independientes mediante el método estándar. En la Tabla 4 se presenta un resumen de los principales resultados.

En el primer modelo de regresión, la variable dependiente fue la dimensión de IP progresista, y los cuatro valores sociales explicaron un $15 \%$ de la varianza $(F(4,84)=39.53, p<.00)$. Posteriormente, se observó la contribución independiente de los predictores mediante los coeficientes de regresión estandarizados. Los valores posmaterialistas $(\beta=.32)$ y valores hedonistas $(\beta=.18)$ realizan una contribución predictiva de forma positiva en el

Tabla 3.

Correlaciones bivariadas entre ideología politica y valores psicosociales

\begin{tabular}{lcccc}
\hline & Valores posmaterialistas & Valores religiosos & Valores hedonistas & Valores materialistas \\
\hline IP progresista & $.19^{* * *}$ & -.09 & $.20^{* * *}$ & $-.11^{* *}$ \\
IP conservadora & $-.12 * * *$ & $.49 * * *$ & -.08 & $.36^{* * *}$ \\
\hline
\end{tabular}

Nota. ${ }^{* *} p<.01 \mathrm{y} * * * p<.000$. 
Tabla 4.

Predicción de las dimensiones de IP a partir de cuatro valores sociales

\begin{tabular}{|c|c|c|c|c|c|c|}
\hline \multirow{2}{*}{$\begin{array}{c}\text { Variable dependiente } \\
\text { Predictor }\end{array}$} & \multicolumn{3}{|c|}{ IP Progresista } & \multicolumn{3}{|c|}{ IP Conservadora } \\
\hline & $\beta$ & $t$ & $p$ & $\beta$ & $t$ & $p$ \\
\hline Valores posmaterialistas & .32 & 8.54 & .00 & -.21 & -6.23 & .00 \\
\hline Valores religiosos & -.03 & -0.80 & .46 & .47 & 14.63 & .00 \\
\hline Valores hedonistas & .18 & 4.71 & .00 & -.04 & -1.25 & .21 \\
\hline Valores materialistas & -.22 & -5.93 & .00 & .19 & 5.59 & .00 \\
\hline$F$ & \multicolumn{3}{|c|}{$(4,84) 39.53 * * *$} & \multicolumn{3}{|c|}{$(4,83) 82.05^{* *}$} \\
\hline$R^{2}$ & \multicolumn{3}{|c|}{.15} & \multicolumn{3}{|c|}{.28} \\
\hline
\end{tabular}

Nota. $* * p<.001 \mathrm{y} * * * p<.000$.

modelo, mientras que los valores materialistas $(\beta=-0.22)$ tienen una contribución predictiva de forma negativa.

Finalmente, en el segundo modelo de regresión, la variable dependiente fue la dimensión de IP conservadora, y los cuatro valores sociales explicaron un $28 \%$ de la varianza $(F(4,83)=82,05, p \leq .00)$; por su parte, los valores religiosos $(\beta=.47)$ y los valores materialistas $(\beta=.19)$ realizan una contribución predictiva de forma positiva en el modelo, mientras que los valores posmaterialistas $(\beta=-0.21)$ hacen una contribución predictiva de forma negativa.

\section{Discusión}

El objetivo de este trabajo fue examinar las propiedades psicométricas de una escala operativa de ideología política utilizando el MEC. Este modelo psicométrico permite asegurar que los parámetros de las personas y de los ítems se expresen en las mismas unidades, y ajustar los datos al modelo demostrando que las personas son independientes de los ítems administrados y que la escala presenta propiedades de intervalo - como es el tipo logit - (Engelhard Jr., 2013). Este aporte es especialmente significativo por la escasez de instrumentos de evaluación que entiendan la IP de un modo operativo, multidimensional y que sean ecológicamente válidos. Además, es uno de los pocos estudios que presenta datos de muestras poblacionales probabilísticas, lo cual incrementa su validez externa.

En líneas generales, los ítems de la escala de IP presentaron propiedades psicométricas aceptables. La unidimensionalidad se corroboró por separado para cada una de las dos dimensiones de IP, luego de que el análisis exploratorio sustentara una estructura bidimensional - progresista y conservadora- Estos resultados difieren parcialmente de estudios anteriores (Brussino et al., 2011), donde se halló un constructo de IP con dos dimensiones del progresismo y dos dimensiones del conservadurismo. Por otro lado, el supuesto de independencia de los ítems se cumplimentó cuando los ítems que no ajustaron a los datos no fueron contemplados en el modelo. Aunque estos no fueron eliminados de la escala de manera definitiva, sería adecuado revisar los contenidos que mide cada uno y valorar si su supresión afecta la validez de contenido del instrumento.

El análisis de las categorías de respuestas ha puesto de manifiesto la posibilidad de reducir el número de estas en cada ítem, dado que las categorías intermedias fueron las que presentaron menores frecuencias. Específicamente, las categorías "En desacuerdo" y "De acuerdo" no representan, en ninguna parte del continuo, la respuesta más probable. Este resultado indica que la respuesta más probable para una persona que supera el primer nivel de ejecución ("Totalmente en desacuerdo") es el tercer nivel de ejecución ("Ni de acuerdo ni en desacuerdo"). Una posible solución a esta situación es utilizar otra etiqueta verbal que sustituya a la categoría "En desacuerdo" y "De acuerdo", o bien eliminarla. En este trabajo, se observó que una vez colapsadas las categorías de respuesta extremas y valorado el comportamiento de los ítems con un modelo de respuesta de tres categorías - en lugar de cinco - se ordenaron las medidas y mejoró el ajuste.

Respecto a la precisión de los ítems, estos han mostrado sus valores máximos en un rango del continúo fijado entre -0.99 y 1.45 logits para la subescala progresista, y entre -1.040 y 0.90 logits para la subescala conservadora; es en este rango del continuo donde el error de medida es inferior. Por el contrario, las posiciones extremas - superior e inferior - del continuo de la IP son las que llevan asociadas un mayor error de medida. Esta es una de las ventajas de los modelos de TRI que permite aportar un error de medida para cada región del continuo. Al respecto, es posible dar solución a estos efectos por medio de la redacción de nuevos ítems que se sitúen en las regiones superior e inferior del continuo. 
Como se mencionó previamente, existen tres ítems de la subescala progresista y tres ítems de la subescala conservadora que no muestran un ajuste aceptable según los parámetros considerados para ello. Entre las posibles explicaciones de la falta de ajuste destacan los siguientes aspectos puntualizados por la literatura especializada: que se trata de ítems que no miden una misma variable, de ahí que se incumpla el supuesto de unidimensionalidad; que las capacidades de discriminación de los ítems no son similares; y que existen problemas con el contenido de los ítems (Engelhard Jr., 2013). Teniendo presentes estas posibilidades, se debería realizar un seguimiento del comportamiento de estos seis ítems en futuras investigaciones.

Los índices de dificultad y los niveles de habilidad de los/as participantes cubrieron gran parte del continúo medido, y los índices de fiabilidad indican que la localización de las personas e ítems sería previsiblemente reproducible. El ajuste global de los ítems fue adecuado en la mayoría de los casos. Especialmente, cuando se eliminaron los ítems de ambas escalas, el ajuste del modelo fue satisfactorio.

La zona de alineamiento entre la dificultad de los ítems y la presencia del rasgo latente en los/as respondientes fue alta, y se observó que entre un $89.39 \%$ (progresista) y un $90.34 \%$ (conservador) de los/as mismos/as respondieron coherentemente a los ítems de la prueba. Por otro lado, en futuras aplicaciones, y pensando en la creación de un banco de ítems, el conocer la localización de los ítems en el continuo de IP permitiría crear tests adaptativos informatizados para medir los diferentes niveles del constructo de interés. Así, no solo sería posible establecer los diferentes niveles de rasgo en IP — conservador o progresista - sino también establecer las diferentes posiciones ante algunas políticas.

Finalmente, como un indicador de validez externa del instrumento, se estudió su relación con los valores sociales, y los resultados fueron consistentes con la evidencia antecedente, de modo tal que fue la dimensión de conservadurismo de la IP la que correlacionó más consistentemente con los valores, especialmente con la importancia atribuida a los valores religiosos y en segundo lugar a los materialistas (Brussino et al., 2013). Por su parte, la dimensión progresista de la IP evidenció un patrón de relaciones relativamente menos consistente, aunque en el sentido esperado, correlacionando positivamente con valores posmaterialistas y hedonistas, y negativamente con los materialistas. En esta línea, el abordaje del o los progresismos sigue siendo un desafío para el campo de la psicología política, lo cual puede responder parcialmente a algunas características cognitivas y psicopolíticas de las personas menos conservadoras. En este sentido, las posiciones progresistas se relacionan con un estilo cognitivo más analítico y una menor rigidez cognitiva o necesidad de cierre cognitivo (Azevedo et al.,
2019), lo cual podría explicar una mayor variabilidad y una consistencia relativamente menor en sus posicionamientos, lo que hace más difícil su medición objetiva.

En conclusión, las propiedades psicométricas de la Escala de IP aún están en estudio, pues se considera que sus ítems tienen un anclaje contextual y político significativo. En este escenario, es recomendable seleccionar los mejores indicadores de esa cultura política, pues no resulta problemático realizar modificaciones en esta instancia del desarrollo del instrumento (Shea et al., 2009).

En línea con lo anterior, es preciso explicitar algunas limitaciones de este estudio que podrían ser resueltas en investigaciones posteriores. En primer lugar, es relevante corroborar la adecuación de una escala de respuesta de tres opciones, tal como fue estudiada aquí. En segundo lugar, los perfiles ideológicos progresistas siguen siendo relativamente más difíciles de captar con una escala, de manera que estudios posteriores podrían avanzar en indagaciones que, a través de técnicas de triangulación metodológica, complementen nuestra comprensión del perfil ideológico y de cultura política de los segmentos progresistas de la sociedad argentina. Finalmente, señalamos antes que una comprensión operativa de la IP debe pensarse necesariamente en su contexto sociopolítico, por lo tanto, es posible que esta escala deba sufrir modificaciones en el tiempo, así como ser revisada en cada contexto de aplicación.

De cualquier modo, la evidencia presentada resulta suficiente para sostener la relevancia de un abordaje operativo de la IP y la adecuación del instrumento desarrollado para ello. Los resultados obtenidos al aplicar estos modelos conducen a una mejor comprensión — desde el punto de vista de la validez - de la construcción que se está explorando (Baghaei, 2008), y nos ha permitido examinar las propiedades psicométricas de la Escala de IP en detalle.

\section{Referencias}

Alonso, D., \& Brussino, S. (2018). Estructura y evidencias de validez de la versión en español del Cuestionario de Valores Psicosociales (QVP-24). Liberabit, 24(2), 213-230. https:// doi.org/10.24265/liberabit.2018.v24n2.04

American Psychological Association [APA]. (2010). Ethical principles of psychologist and code of conduct. APA.

Andrich, D. (1978). Application of a psychometric rating model to ordered categories which are scored with successive integers. Applied Psychological Measurement, 2(4), 581-594. https://doi.org/10.1177/014662167800200413

Andrich, D., Sheridan, B., \& Luo, G. (2010). Rasch models for measurement: RUMM2030. RUMM Laboratory Pty Ltd. 
Azevedo, F., Jost, J. T., Rothmund, T., \& Sterling, J. (2019). Neoliberal Ideology and the Justification of Inequality in Capitalist Societies: Why Social and Economic Dimensions of Ideology Are Intertwined. Journal of Social Issues, 75, 49-88. https://doi.org/10.1111/josi.12310

Baghaei, P. (2008). The Rasch model as a construct validation tool. Rasch Measurement Transaction, 22(1), 1145-1146. https://www.rasch.org/rmt/rmt221a.htm\#: :text=In $\% 20$ $1955 \% 2$ C $\% 20$ Cronbach $\% 20$ and $\% 20$ Meehl, being $\% 20$ the $\% 20$ most $\% 20$ important $\% 20$ one

Barros, T. S., Torres, A. R. R., \& Pereira, C. (2009). Autoritarismo e adesão a sistemas de valores psicossociais. PsicoUSF, 14(1), 47-57. https://doi.org/10.1590/ s1413-82712009000100006

Bauer, P. C., Barberá, P., Ackermann, K., \& Venetz, A. (2017). Is the left-right scale a valid measure of ideology? Political Behavior, 39(3), 553-583. https://doi.org/10.1007/ s11109-016-9368-2

Brussino, S., Imhoff, D., Paz García, A. P., \& Dreizik, M. (2017). El análisis psico-político de la ideología política. En Brussino, S. (Coord.), Políticamente, contribuciones desde la psicología política en Argentina (pp. 73-104). CONICET. https://rdu.unc.edu.ar/handle/11086/4910

Brussino, S., Imhoff, D., Rabbia, H. H., \& Paz García, A. P. (2013). Ideología política en torno a issues y valores sociales: un estudio correlacional en ciudadanos de Córdoba Argentina. América Latina Hoy, 65, 161-182. https://doi. org/10.14201/alh201365161182

Brussino, S., Rabbia, H. H., Imhoff, D., \& Paz García, A. P. (2011). Dimensión Operativa de la Ideología Política en ciudadanos de Córdoba/Argentina. Revista Psicología Politica, 43, 85-106. https://www.uv.es/garzon/psicolo gia\%20politica/N43-4.pdf

Caprara, G. V., Schwartz, S., Capanna, C., Vecchione, M., \& Barbaranelli, C. (2006). Personality and politics: Values, traits, and political choice. Political Psychology, 27(1), 1-28. https://doi.org/10.1111/j.1467-9221.2006.00447.x

Caprara, G. V., Vecchione, M., Schwartz, S. H., Schoen, H., Bain, P., Silvester, J., Cieciuch, J., Pavlopoulos, V., \& Bianchi, C. (2017). Basic values, ideological selfplacement, and voting: A cross-cultural study. Cross-Cultural Research, 51(4), 388411. https://doi.org/10.1177/1069397117712194

Caprara, G. V., Vecchione, M., Schwartz, S. H., Schoen, H., Bain, P. G., Silvester, J., ., \& Caprara, M. G. (2018). The contribution of religiosity to ideology: Empirical evidences from five continents. Cross-Cultural Research, 52(5), 524541. https://doi.org/10.1177/1069397118774233

Cohen, J. (1988). Statistical power analysis for the behavioral sciences (2. ${ }^{\mathrm{a}}$ ed.). Erlbaum.

Cordero García, G. (Noviembre, 2008). ¿Qué es ideología? El caso español. Trabajo presentado en el Seminario de Investigadores en Formación del Departamento de Ciencia Política de la Universidad Autónoma de Madrid. Madrid, España. https://doi.org/10.14195/978-989-26-0319-3_39
Costello, T. H., \& Lilienfeld, S. O. (2020). Social and economic political ideology consistently operate as mutual suppressors: implications for personality, social, and political psychology. Social Psychological and Personality Science, pre-print. https://doi.org/10.1177/1948550620964679

Crawford, J. T., Brandt, M. J., Inbar, Y., Chambers, J. R., \& Motyl, M. (2017). Social and economic ideologies differentially predict prejudice across the political spectrum, but social issues are most divisive. Journal of Personality and Social Psychology, 112(3), 383. https://doi.org/10.31234/ osf.io/3g5pz

D’Adamo, O., \& García Beaudoux, V. (2002). Actitudes políticas. En F. Morales, D. Páez, Kornblit, A. L., \& D. Asún (Coord.), Psicología Social. Pearson Educación.

Engelhard Jr., G. (2013). Invariant measurement: Using Rasch models in the social, behavioral, and health sciences. Routledge. https://doi.org/10.4324/9780203073636

Federico, C. M., \& Schneider, M. C. (2007). Political expertise and the use of ideology: Moderating effects of evaluative motivation. Public Opinion Quarterly, 71(2), 221-252. https://doi.org/10.1093/poq/nfm010

Ferrando, P. J., \& Lorenzo-Seva, U. (2016). A note on improving EAP trait estimation in oblique factor-analytic and item response theory models. Psicológica, 37(2), 235-247. https://www.uv.es/revispsi/articulos2.16/7Ferrando.pdf

Freire, A., \& Kivistik, K. (2013). Mapping and explaining the use of the left-right divide. Brazilian Political Science Review, 7(3), 61-89. https://doi.org/10.1590/ s1981-38212013000300003

Glutting, J. (2002). Some psychometric properties of a system to measure ADHD among college students. Measurement and Evaluation in Counselling and Development, 34, 194209. https://doi.org/10.1080/07481756.2002.12069037

Jost, J. T. (2006). The end of the end of ideology. American Psychologist, 61(7), 651-670. https://doi. org/10.1037/0003-066x.61.7.651

Kalmoe, N. P. (2020). Uses and abuses of ideology in political psychology. Political Psychology, 41(4), 771-793. https:// doi.org/10.1111/pops. 12650

Laméris, M. D., Jong-A-Pin, R., \& Garretsen, H. (2018). On the measurement of voter ideology. European Journal of Political Economy, 55, 417-432. https://doi.org/10.1016/j. ejpoleco.2018.03.003

Lau, R. R., \& Redlawsk, D. P. (2006). How voters decide: Information processing in election campaigns. Cambridge University Press. https://doi.org/10.1017/ cbo9780511791048.001

Lohr, S. (2000). Muetreo: Diseño y Análisis (1. a ed.). International Thomson.

Lorenzo-Seva, U., \& Ferrando, P. J. (2006). FACTOR: A computer program to fit the exploratory factor analysis model. Behavioral Research Methods, Instruments and Computers, 38(1), 88-91. https://doi.org/10.3758/BF03192753 
Lorenzo-Seva, U., \& Ferrando, P. J. (2019). Robust Promin: un método para la rotación de factores de diagonal ponderada. Liberabit, 25(1), 99-106. https://doi.org/10.24265/libera bit.2019.v25n1.08

McCoy, J., Rahman, T., \& Somer, M. (2018). Polarization and the global crisis of democracy: Common patterns, dynamics, and pernicious consequences for democratic policies. American Behavioral Scientist, 62(1), 16-42. https://doi. org/10.1177/0002764218759576

McDonald, R. P. (1999). Test theory: A unified treatment. Lawrence Erlbaum Associates, Inc.

Montero, I., \& León, O. G. (2007). A guide for naming research studies in Psychology. International Journal of clinical and Health psychology, 7(3), 847-862. http://www.aepc.es/ij chp/articulos_pdf/ijchp-256.pdf

Morgan, G. S., \& Wisneski, D. C. (2017). The structure of political ideology varies between and within people: Implications for theories about ideology's causes. Social Cognition, 35(4), 395-414. https://doi.org/10.1521/soco.2017.35.4.395

Morrison, A. P., Wells, A., \& Nothard, S. (2000). Cognitive factors in predisposition to auditory and visual hallucinations. British Journal of Clinical Psychology, 39, 67-78. https:// doi.org/10.1348/014466500163112

Pereira, C., Camino, L., \& Bastos da Costa, J. (2004). Análise fatorial confirmatória do Questionário de Valores Psicossociais-QVP24. Estudos de Psicologia, 9(3), 505512. https://doi.org/10.1590/s1413-294x2004000300013

Pereira, C., Camino, L., \& Bastos da Costa, J. (2005). Um estudo sobre a integração dos níveis de análise dos sistemas de valores. Psicologia: reflexão e crítica, 18(1), 16-25. https:// doi.org/10.1590/s0102-79722005000100004

Piurko, Y., Schwartz, S. H., \& Davidov, E. (2011). Basic personal values and the meaning of left-right political orientations in 20 countries. Political Psychology, 32(4), 537-561. https://doi.org/10.1111/j.1467-9221.2011.00828.x
Schwartz, S. H. (2003). A proposal for measuring value orientations across nations. En Questionnaire Package of the European Social Survey (pp. 259-290). http:// www.europeansocialsurvey.org/index.php?option=com docman\&task $=$ doc_view\&gid $=126 \&$ Itemid $=80$

Shea, B. J., Wells, G. A., Bouter, L. M., Kristjansson, E., Grimshaw, J., Henry, D. A., \& Boers, M. (2009). AMSTAR is a reliable and valid measurement tool to assess the methodological quality of systematic reviews. Journal of Clinical Epidemiology, 62(10), 1013-1020. https://doi. org/10.1016/j.jclinepi.2008.10.009

Sheldon, K. M., \& Nichols, C. P. (2009). Comparing democrats and republicans on intrinsic and extrinsic values. Journal of Applied Social Psychology, 39(3), 589-623. https://doi. org/10.1111/j.1559-1816.2009.00452.x

Smith, E. V. (2002). Detecting and evaluating the impact of multidimensionality using item fit statistics and principal component analysis of residuals. Journal of Applied Measurement, 3(2) 205-231.

Steinmetz, H., Isidor, R., \& Baeuerle, N. (2012). Testing the circular structure of human values: A meta-analytical structural equation modelling approach. Survey Research Methods, 6(1), 61-75. https://doi.org/10.18148/srm/2012.v6i1.5096

Tabachnick, B. G., \& Fidell, L.S. (2001). Using Multivariate Statistics, (4. ${ }^{\circ}$ ed.). Allyn \& Bacon.

Tennant, A., \& Conaghan, P. G. (2007) The Rasch measurement model in rheumatology: what is it and why use it? When should it be applied and what should one look for in a Rasch paper? Arthritis Rheum, 57(8), 1358-1362. https:// doi.org/10.1002/art.23108

Tennant, A., \& Pallant, J. F. (2006) Unidimensionality matters! (A tale of two Smiths?). Rasch Measurement Transactions, 20, 1048-1051. https://www.rasch.org/rmt/rmt201c.htm 
Ítems de la subescala de progresismo

1. Se debería despenalizar la tenencia y cultivo de marihuana para consumo personal.

5. El Estado debería hacer consultas populares vinculantes antes de tomar grandes decisiones para el destino del país.

6. El Estado debería preservar el sistema de fondos de pensiones (jubilaciones) como un sistema eminentemente público.

9. Los servicios públicos esenciales (agua, luz, gas) deberían ser propiedad del Estado.

11. El Estado debería promover campañas de concientización sobre el consumo responsable de sustancias como la marihuana.

13. El Estado debería garantizar condiciones de trabajo dignas y saludables para los/as trabajadores/as sexuales (prostitutos/as).

16. El Estado debería otorgarle tierras a las comunidades indígenas que habitan en el país para que puedan autogobernarse.

17. En todas las escuelas se debería enseñar un idioma de nuestros pueblos indígenas para aprender a apreciar su herencia cultural.

20. El Estado debería garantizar un ingreso mínimo a todos los niños sin importar la situación laboral de sus padres.

21. El Estado debería garantizar que todos los ciudadanos accedan por igual al teatro, cine y demás producciones culturales locales.

24. Está bien que desocupados y vecinos realicen piquetes y cortes de calles o rutas, ya que es la única manera que tienen para presionar y lograr que sus reclamos sean atendidos por los gobiernos

25. El Estado debería hacer lo posible por evitar la concentración de medios de comunicación en pocas manos, y asegurar asi la pluralidad de expresión.

26. El Estado debería autorizar el consumo de marihuana en enfermos terminales.

27. Cuando hay crisis económica, el Estado debería aumentar el gasto en programas de asistencia social y subsidios (como el programa nacional "jefas y jefes de hogar").

28. La propiedad de la tierra debe ser de quien la trabaje

31. Está bien que se invierta parte del presupuesto nacional en medios masivos de comunicación de propiedad estatal.
Items de la subescala de conservadurismo

2. Los hombres y mujeres homosexuales no deberían bajo ningún pretexto ser autorizados a adoptar hijos.

3. El aborto es un crimen y debe ser perseguido y penado por la justicia en todas las circunstancias.

4. La ley no debería permitir a personas homosexuales dar clases en las escuelas.

7. A veces un gobierno militar puede ser preferible a uno democrático.

8. Sólo los padres tienen derecho a enseñar a sus hijos temas relacionados con la sexualidad; el colegio no debería intervenir en estas cuestiones.

10. El Estado debería asegurar más policías en la calle para el control del crimen y la delincuencia, aún si para ello fuera necesario recortar el presupuesto de otras áreas importantes como trabajo, salud y educación.

12. Los políticos deberían escuchar más los reclamos y propuestas de la Iglesia Católica sobre los problemas que atraviesa la sociedad.

14. La pena de muerte es una medida que ayuda a bajar la delincuencia.

15. El Estado debería prohibir la exhibición pública de obras de arte que sean ofensivas a la moral o religión de determinados grupos de personas.

18. Se debería detener por "apología del delito" a aquellos jóvenes que utilicen ropas estampadas con referencias favorables al consumo de drogas.

19. La educación sexual en jóvenes es peligrosa porque los motiva a una iniciación sexual temprana.

22. Está bien que el Estado sostenga económicamente a la Iglesia Católica.

23. Ante la crisis económica, nuestro país debería ser menos permisivo con el ingreso de inmigrantes que compiten con los ciudadanos locales en la búsqueda de trabajo y mejores condiciones de vida.

29. El Estado debería privatizar todas las empresas públicas ineficientes.

30. No deberían utilizarse los medios de comunicación estatal para publicidad oficial o propaganda gubernamental. 